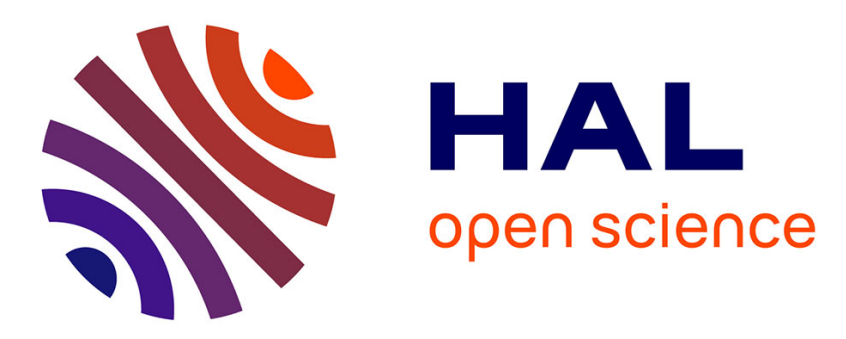

\title{
Continuous Closed Form Trajectories Generation and Control of Redundantly Actuated Parallel Kinematic Manipulators
}

Moussab Bennehar, Ahmed Chemori, Sébastien Krut, François Pierrot

\section{To cite this version:}

Moussab Bennehar, Ahmed Chemori, Sébastien Krut, François Pierrot. Continuous Closed Form Trajectories Generation and Control of Redundantly Actuated Parallel Kinematic Manipulators. SSD: Systems, Signals and Devices, Feb 2014, Castelldefels-Barcelona, Spain. 10.1109/SSD.2014.6808879 . lirmm-00947459

\section{HAL Id: lirmm-00947459 \\ https://hal-lirmm.ccsd.cnrs.fr/lirmm-00947459}

Submitted on 16 Feb 2014

HAL is a multi-disciplinary open access archive for the deposit and dissemination of scientific research documents, whether they are published or not. The documents may come from teaching and research institutions in France or abroad, or from public or private research centers.
L'archive ouverte pluridisciplinaire HAL, est destinée au dépôt et à la diffusion de documents scientifiques de niveau recherche, publiés ou non, émanant des établissements d'enseignement et de recherche français ou étrangers, des laboratoires publics ou privés. 


\title{
Continuous Closed Form Trajectories Generation and Control of Redundantly Actuated Parallel Kinematic Manipulators
}

\author{
Moussab Bennehar*, Ahmed Chemori*, Sbastien Krut* and Franois Pierrot* \\ ${ }^{*}$ Robotics Department, LIRMM, Univ. Montpellier 2 - CNRS \\ 161 rue Ada, 34090 Montpellier, France. \\ Email: bennehar(chemori, krut, pierrot)@lirmm.fr
}

\begin{abstract}
This paper deals with continuous closed form trajectories generation and control of redundantly actuated parallel kinematic manipulators. Continuous closed form trajectories are usually given by means of analytical functions of time and show inherent discontinuities in velocity and/or acceleration profiles. In order to solve this problem, we propose an approach consisting of modifying the time function without changing the overall closed shape of the trajectory in the operational space. To deal with the actuation redundancy, an extended version of the PD controller with computed feedforward is proposed. The resulting control torques are projected using a regularization matrix in order to remove internal forces that may be dangerous for the manipulator. The proposed trajectory generator as well as the controller are implemented in real-time on Dual-V; a redundantly actuated parallel manipulator developed in our laboratory.
\end{abstract}

\section{INTRODUCTION}

Parallel Kinematic Manipulators (PKMs) are mostly known for their better performances over their serial counterparts [9]. Indeed, in contrast with the serial ones, the actuators of PKMs are located on the fixed base and, hence, result in a very lightweight moving mass [3]. Furthermore, the closed kinematic chains yield to a stiffer and more accurate structure. Nevertheless, PKMs exhibits many drawbacks that moderate their expansion in industrial applications. The abundance of singularities and the limited workspace are the most noteworthy ones. While the latter is a matter of optimal design, the former can be solved by actuation redundancy. Hence, Redundantly Actuated PKMs (RA-PKMs) are increasingly attracting more interest of researchers in the last two decades.

Actuation redundancy significantly improves the performance of PKMs by homogenizing the physical properties throughout the workspace, allowing the manipulator to achieve higher accelerations. However, despite the aforementioned qualities, PKMs potential is not yet completely explored and is still an open research field. From one hand, mechanical design, identification and optimization can be further investigated in order to increase their acceptance in industrial applications. From the other hand, the close-loop constraints and the inherent nonlinearities give rise to more challenging tasks in terms of trajectory generation and control.

This research was supported by the French National Research Agency, within the project ANR-ARROW
Thanks to their high dynamic performances, PKMs are typically used for industrial high-speed applications such as pick-and-place in food industry [10]. Hence, most researches in terms of trajectory generation focused only on classical Point-to-Point (PtP) trajectories [5], [4], [11]. Nevertheless, the inherent stiffness and accuracy of PKMs awarded them to be used in more complex industrial tasks such as laser cutting [12] or machining [13]. In this case, Continuous Closed Shape (CCS) trajectories draw more attention than traditional PtP ones. This class of trajectories, however, has not been sufficiently investigated in the literature.

It is conventional that PKMs share many physical properties with serial robots. Consequently, most of the control literature developed for serial manipulators has been straightforwardly implemented on PKMs [14], [15]. However, in the case of RA-PKMs, a particular property characterizing this class of manipulators needs a special attention. The nonuniqueness of the inverse dynamics solution yields to control forces with no effects on the moving platform of the robot [6]. These control forces produce undesired internal pre-stress in the kinematic chains that can harm the manipulator and cause a loss of energy. Consequently, the control scheme should take into consideration such a phenomenon.

In this work, we address the two aforementioned problems, namely; the CCS trajectories generation and the reduction of internal forces for RA-PKMs. We propose a trajectory generator that takes into consideration continuity constraints, both in task and joint spaces. As regards to the control solution, a joint space PD with computed feedforward controller [16] is proposed. This control scheme has the advantage of being lightweight in terms of computation time while compensating some of the nonlinearities. In order to deal with the internal forces issue, the proposed controller is extended with a projection of the generated control torques with the regularization. The proposed approach is then validated in real-time on a 3 dof RA-PKM, namely, the Dual-V.

This paper is organized as follows. In section 2, the dynamic model of the Dual-V robot is presented. Section 3 is devoted to the proposed trajectory generator. The proposed control solution is detailed in Section 4. Experimental results are presented in Section 5. Finally, conclusions and perspectives are drawn in Section 6. 


\section{DYNAMIC MODELING OF THE DUAL-V ROBOT}

Dual-V is a 3 dof planar redundantly actuated parallel manipulator belonging to the 4-RRR family. The arrangement of the four chains allows three independent movements for its end-effector (a traveling plate): two translations in the plane and one rotation about the $z$-axis. Hence, the Cartesian coordinates the traveling plate are described by the vector $X=[x, y, \theta]^{T}$. For the dynamic modeling of the robot, the approach developed in [3] has been extended to take into account the rotational inertia of the forearms.

The required torque to move the robot's mechanical structure can be decomposed into three sub-torques, namely; $\tau_{1}, \tau_{2}, \tau_{3} \in \mathbb{R}^{4}$. Each sub-torque is responsible for the movement of a part of the structure. The torque $\tau_{1}$ is the required torque to move the traveling plate and a part of the mass of the forearms, it can be calculated using the equations of power of the actuators as follows:

$$
\tau_{1}=J_{m}^{T *} M_{I} \ddot{X}
$$

where $M_{I} \in \mathbb{R}^{3 \times 3}$ is the mass matrix of the concerned part of the links to be moved in Cartesian space, $J_{m}^{T *}=J_{m}\left(J_{m}^{T} J_{m}\right)^{-1} \in$ $\mathbb{R}^{4 \times 3}$ is the pseudo-inverse of the transpose of the inverse Jacobian matrix $J_{m}$ and $\ddot{X} \in \mathbb{R}^{3}$ is the acceleration vector of the traveling plate. The component $\tau_{2}$ is the required torque to move the arms, the counter-masses and the remaining part of the couplers, it can be expressed as:

$$
\tau_{2}=M_{I I} \ddot{q}=M_{I I}\left(\dot{J}_{m} \dot{X}+J_{m} \ddot{X}\right)
$$

where $M_{I I} \in \mathbb{R}^{4 \times 4}$ is the mass matrix regrouping dynamic parameters of the involved parts of the structure in joint space, $\dot{X} \in \mathbb{R}^{4}$ is the traveling plate velocity vector and $\dot{J}_{m}$ is the time derivative of the inverse Jacobian matrix. Up to now, only the inertia of the two mass model [8] of the couplers is considered. Though this is a righteous hypotheses when light material is used, it fails when the links are made with heavy material such as steal or aluminum. This is the case of the Dual-V robot and, hence, an additional term should be added. The torque term $\tau_{3}$ is the additional torque component to compensate for the real inertia of the couplers and that of the equivalent mass model. Due to the number of pages limitation, the details of this additional term are not provided herein and the reader is referred to [8] for a full description of the Dual-V robot dynamic modeling. It is worth noting though that $\tau_{3}(X, \dot{X}, \ddot{X})$ is a highly nonlinear function with respect to its arguments.

Finally, the full dynamic model of the Dual-v is written by regrouping the three sub-torques as follows:

$$
\tau=J_{m}^{T *} M_{I} \ddot{X}+M_{I I}\left(\dot{J}_{m} \dot{X}+J_{m} \ddot{X}\right)+\tau_{3}(X, \dot{X}, \ddot{X})
$$

The CAD model of the Dual-V with parameters definition is depicted in Fig. 1 and its geometric and dynamic parameters are summarized in Table I. Further details about the Dual-V robot are given in [7] and [2].

\begin{tabular}{|c|c|c|c|}
\cline { 2 - 4 } \multicolumn{1}{c|}{} & Length $[\mathrm{m}]$ & Mass $[\mathrm{Kg}]$ & Inertia $\left[\mathrm{Kgm}^{2}\right]$ \\
\hline Crank & 0.2800 & 1.169 & 0.012967 \\
\hline Coupler & 0.2800 & 0.606 & 0.006417 \\
\hline Traveling plate & 0.22 & 0.899 & 0.008168 \\
\hline
\end{tabular}

TABLE I: Dual-V geometric and dynamic parameters

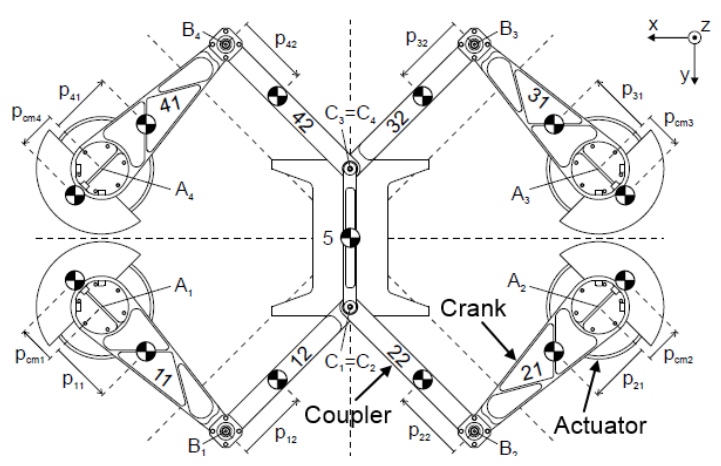

Fig. 1: The CAD of the Dual-V with parameters definition

\section{REFERENCE TRAJECTORIES GENERATION}

CCS trajectories are more complex than traditional PtP ones [4],[5] (e.g pick-and-place trajectories) and many of them are generated by means of analytical functions parametrized by the time variable $t$. Therefore, generating trajectories with continuity constraints on velocities and accelerations may not be possible using their original analytical equations. Continuity of trajectories is a crucial constraint for the robot drives since discontinuities in the trajectories can generate discontinuous control torques and thus, lead to undesired behavior of the mechanical structure such as vibrations or instabilities.

In this paper we are interested in the class of trajectories described by a sum of weighted sin and cosine functions. This class of trajectories has been chosen because it covers a large amount of simple and complex geometrical shapes (e.g. circles, ellipses, deltoids, ... ). The general analytical form of the trajectories in question is given by:

$$
x(t)=\sum_{i=1}^{n} a_{i} \cos ^{\alpha_{i}}\left(2 \pi \frac{n_{1 i}}{T} t\right)+b_{i} \sin ^{\beta_{i}}\left(2 \pi \frac{n_{2 i}}{T} t\right)
$$

where $T \in \mathbb{R}^{+}$is the trajectory duration and $a_{i}, \alpha_{i}, b_{i}, \beta_{i}, n_{j i} \in$ $\mathbb{R} ; i=1, \ldots, n ; j=1,2$ are the parameters defining the shape of the trajectory.

In the sequel, we will first give an illustrative example of direct application of (4). Then, its major drawback is highlighted and a solution to overcome it is proposed.

\section{A. Classical method}

Let $x_{d}(t)$ be the desired Cartesian position defined by (4) to be tracked by the traveling plate of the robot. These desired trajectories are fully included in the workspace of the robot away from singularities. The desired velocity and acceleration trajectories for this motion are obtained by differentiating $x_{d}(t)$ with respect to time, thus: 


$$
\begin{gathered}
v_{d}(t)=-\frac{2 \pi}{T} \sum_{i=1}^{n} a_{i} \alpha_{i} n_{1 i} \sin ^{\alpha_{i}-1}\left(2 \pi \frac{n_{1 i}}{T} t\right)- \\
b_{i} \beta_{i} n_{2 i} \cos ^{\beta_{i}-1}\left(2 \pi \frac{n_{2 i}}{T} t\right) \\
a_{d}(t)=-\left(\frac{2 \pi}{T}\right)^{2} \sum_{i=1}^{n} a_{i} \alpha_{i}\left(\alpha_{i}-1\right) n_{1 i}^{2} \cos ^{\alpha_{i}-2}\left(2 \pi \frac{n_{1 i}}{T} t\right) \\
+b_{i} \beta_{i}\left(\beta_{i}-1\right) n_{2 i}^{2} \sin ^{\beta_{i}-2}\left(2 \pi \frac{n_{2 i}}{T} t\right)
\end{gathered}
$$

Without loss of generality, assume that the robot starts and finishes its movement with a null velocity and a null acceleration (i.e. $v(0)=v(T)=0$ and $a(0)=a(T)=0$ ). Using the original analytical functions (5) and (6), however, may lead to non-zero initial velocity and/or acceleration. Indeed, if we replace $t=0$ or $t=T$ in (5) and (6) we will obtain values dependent on initial and final conditions and thus, does not necessarily equal zero. For instance, the obtained initial conditions are given by:

$$
\begin{array}{r}
v_{d}(0)=-\frac{2 \pi}{T} \sum_{i=1}^{n} b_{i} \beta_{i} n_{2 i}=f\left(b_{i}, \beta_{i}, n_{2 i}\right) \\
a_{d}(0)=-\left(\frac{2 \pi}{T}\right)^{2} \sum_{i=1}^{n} a_{i} \alpha_{i}\left(\alpha_{i}-1\right) n_{1 i}^{2}=f\left(a_{i}, \alpha_{i}, n_{1 i}\right)
\end{array}
$$

therefore, the resulting trajectories can be velocity and/or acceleration discontinuous leading to discontinuous control torques and therefore big tracking errors or even instability.

To further illustrate this inconvenience let's consider a simple circular trajectory with a radius $r$ and a center whose coordinates are $\left(x_{c}, y_{c}\right)=(0,0)$. The resulting trajectory needs to be $C^{2}$ continuous (in velocities and accelerations) both in operational and joint spaces in order to be appropriately tracked by the traveling plate. The analytical equations of positions, velocities and accelerations are given by:

$$
\begin{gathered}
\left\{\begin{array}{l}
x_{d}(t)=r \cos \left(\frac{2 \pi}{T}\left(t-t_{0}\right)\right) \\
y_{d}(t)=r \sin \left(\frac{2 \pi}{T}\left(t-t_{0}\right)\right)
\end{array}\right. \\
\left\{\begin{array}{l}
\dot{x}_{d}(t)=-r \frac{2 \pi}{T} \sin \left(\frac{2 \pi}{T}\left(t-t_{0}\right)\right) \\
\dot{y}_{d}(t)=r \frac{2 \pi}{T} \cos \left(\frac{2 \pi}{T}\left(t-t_{0}\right)\right)
\end{array}\right. \\
\left\{\begin{array}{l}
\ddot{x}_{d}(t)=-r\left(\frac{2 \pi}{T}\right)^{2} \cos \left(\frac{2 \pi}{T}\left(t-t_{0}\right)\right) \\
\ddot{y}_{d}(t)=-r\left(\frac{2 \pi}{T}\right)^{2} \sin \left(\frac{2 \pi}{T}\left(t-t_{0}\right)\right)
\end{array}\right.
\end{gathered}
$$

These trajectories satisfy equations (4), (5) and (6) being only shifted in time by $t_{0}$. Fig. 2 illustrates the plots of the obtained trajectories using (9), (10) and (11) $\left(t_{0}=0.5 \mathrm{~s}, T=\right.$ $1 s)$. One can notice the presence of discontinuities on both velocity and acceleration profiles that have to be removed. In what follows, we propose a novel method to overcome this drawback and generate $C^{2}$ continuous reference trajectories.
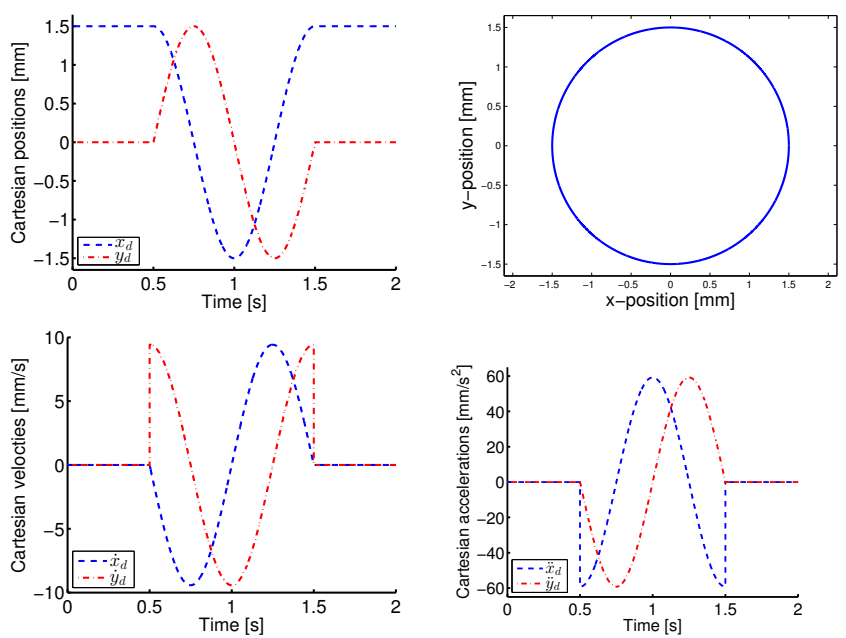

Fig. 2: View of the generated Cartesian trajectories using the classical method

\section{B. Proposed solution: continuous trajectories with a velocity trapezoidal profile}

In order to overcome the discontinuity problem in the velocity and acceleration trajectories, we propose to revisit the general form of the desired operational trajectories. Consider again the previous illustrative example of a circular trajectory. If we check the previous equations of the trajectory, it can be seen that they can be rewritten as follows:

$$
\left\{\begin{array}{l}
x_{d}(t)=r \cos (\lambda(t)) \\
y_{d}(t)=r \sin (\lambda(t))
\end{array}\right.
$$

With $\lambda(t)=\frac{2 \pi}{T}\left(t-t_{0}\right)$ which is a simple affine function of time $t$ and when implemented, does not satisfy any continuity constraint on the velocity and acceleration trajectories. The corresponding velocities and accelerations are obtained by differentiating (12) with respect to time, which gives:

$$
\begin{gathered}
\left\{\begin{array}{l}
\dot{x}_{d}(t)=-r \dot{\lambda}(t) \sin (\lambda(t)) \\
\dot{y}_{d}(t)=r \dot{\lambda}(t) \cos (\lambda(t))
\end{array}\right. \\
\left\{\begin{array}{l}
\ddot{x}_{d}(t)=-r\left[\ddot{\lambda}(t) \sin (\lambda(t))+\dot{\lambda}^{2}(t) \cos (\lambda(t))\right] \\
\ddot{y}_{d}(t)=r\left[\ddot{\lambda}(t) \cos (\lambda(t))-\dot{\lambda}^{2}(t) \sin (\lambda(t))\right]
\end{array}\right.
\end{gathered}
$$

From (13) and (14) one can notice that if $\lambda(t)$ is chosen such that it satisfies the following boundary constraints:

$$
\left\{\begin{array}{l}
\lambda\left(t_{0}\right)=0 ; \quad \dot{\lambda}\left(t_{0}\right)=0 ; \quad \ddot{\lambda}\left(t_{0}\right)=0 \\
\lambda\left(t_{0}+T\right)=2 \pi ; \quad \dot{\lambda}\left(t_{0}+T\right)=0 ; \quad \ddot{\lambda}\left(t_{0}+T\right)=0
\end{array}\right.
$$

one can ensure the continuity on the velocity and acceleration trajectories. One possible solution would then be to choose $\lambda(t)$ with a trapezoidal profile on velocity. Indeed, this choice allows to specify boundary conditions and hence, 

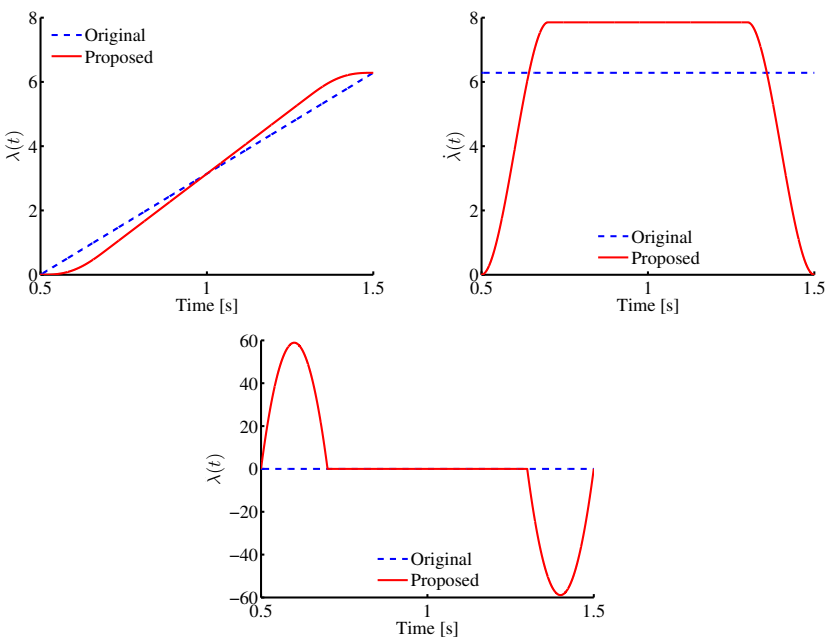

Fig. 3: Evaluation of $\lambda(t), \dot{\lambda}(t)$ and $\ddot{\lambda}(t)$ : original (dashed line) and modified (solid line)

the constraints (15) can be satisfied. A comparison between trapezoidal and linear velocity profile is depicted in Fig. 3. The analytical equations of $\lambda(t)$ are now expressed as:

$$
\left\{\begin{array}{l}
\lambda(t)=\frac{\lambda_{f}-\lambda_{0}}{2 \tau^{3}(T-\tau)}\left[-t^{4}+2 \tau t^{3}\right]+\lambda_{0} ; \quad t_{0} \leq t \leq \tau \\
\lambda(t)=\frac{\lambda_{f}-\lambda_{0}}{(T-\tau)}\left[t-\frac{\tau}{2}\right]+\lambda_{0} ; \quad \tau \leq t \leq T-\tau \\
\lambda(t)=\frac{\lambda_{f}-\lambda_{0}}{2 \tau^{3}(T-\tau)}\left[(t-T)^{4}+2 \tau(t-T)^{3}\right]+\lambda_{f} ; \quad \text { else }
\end{array}\right.
$$

where $\tau$ is the duration of initial acceleration and final deceleration phases that has to be chosen according to the robot dynamic capabilities. $\lambda_{0}$ and $\lambda_{f}$ are the initial and final values respectively for $\lambda(t)$. For instance, in the case of the above circular trajectory example, $\lambda_{0}=0$ and $\lambda_{f}=2 \pi$.

The obtained trajectories using the proposed strategy are now continuous in velocity and acceleration and result in the same trajectory in operational space as the original one (cf. Fig. 4). Therefore, they can be safely used in the control scheme without risks on the actuators.

\section{PROPOSED CONTROL SOLUTION}

It has been shown that PKMs exhibit many similarities with their serial counterparts [1]. Consequently, many control strategies that were mainly developed for serial manipulators have been successfully applied on PKMs. However, in the particular case of RA-PKMs, we notice the existence of antagonistic control torques not affecting the motion of the manipulator. Thus, using conventional control strategies will certainly give occurrence to internal forces (incompatible with the robot's kinematics) [6] and hence, create internal prestress in the mechanism. The antagonistic forces can cause a multitude of undesirable phenomena such as the loss of energy, instability and vibrations. Consequently, the control architecture has to take in consideration this peculiarity.

\section{A. Joint space PD controller}

Consider a RA-PKM with $m$ degrees of freedom and $n>m$ actuators and let the joints position vector be denoted by $q \in$
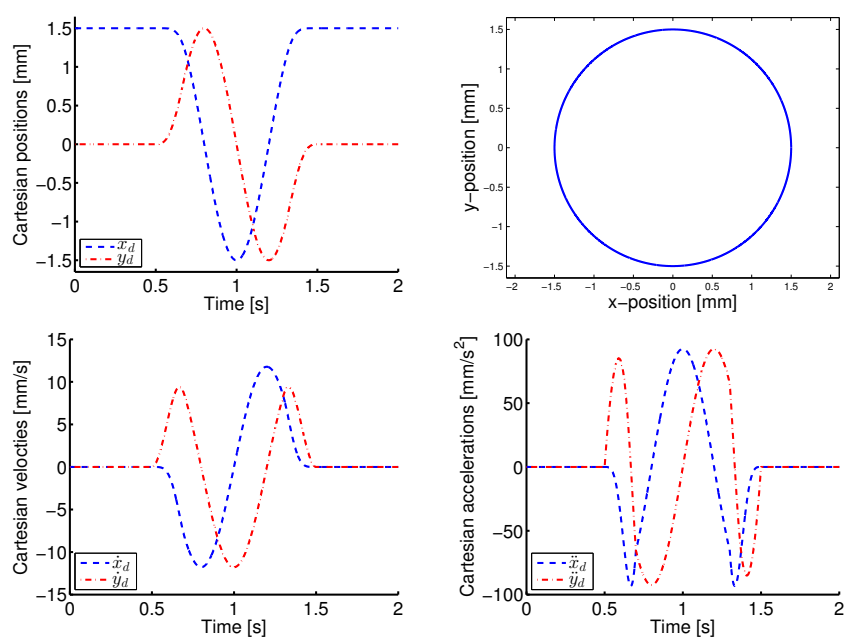

Fig. 4: Obtained $C^{2}$ continuous operational trajectories

$\mathbb{R}^{n}$. The PD control is a decentralized uncoordinated control strategy relying on the measured error between the desired and the actual position. Let $e(t)$ be the joints error vector, i.e : $e(t)=q_{d}(t)-q(t)$, where $q_{d}(t)$ denotes the desired joint trajectory. Then, the PD control action is expressed by:

$$
\tau_{P D}=K_{p} e(t)+K_{d} \dot{e}(t)
$$

where $K_{p}$ and $K_{d}$ are positive definite feedback gain matrices usually chosen diagonal. If the actuators are all identical, which is usually the case for PKMs, the same feedback gains could be selected for each axis, namely; $K_{p}=k_{p} I_{n \times n}$ and $K_{d}=k_{d} I_{n \times n}$, where $I_{n \times n}$ is an identity matrix and $k_{p}$ and $k_{d}$ are positive scalars denoting the feedback gains that should be tuned to achieve satisfactory closed-loop performance. Good accuracy on high accelerations is mostly required in parallel robots.

\section{B. Desired compensation by nonlinear feedforward}

If the inverse dynamics of the robots are well known, it would be interesting to exploit this knowledge to further improve the tracking performance. Indeed, the tracking errors can be significantly improved by compensating the inherent nonlinearities. One possible way would be the addition of a feedforward term. This strategy enables in partially compensating the nonlinear dynamics in the feedforward way, i.e. only desired values of positions, velocities and accelerations are fed to the inverse dynamics model. For the case of the Dual$\mathrm{V}$ robot, the inverse dynamics are given by (3), hence, the feedforward torque is computed as:

$$
\tau_{f f}=J_{m}^{T *} M_{I} \ddot{X}_{d}+M_{I I}\left(\dot{J}_{m} \dot{X}_{d}+J_{m} \ddot{X}_{d}\right)+\tau_{3}\left(X_{d}, \dot{X}_{d}, \ddot{X}_{d}\right)
$$

where the subscript $d$ refers to the desired quantities.

\section{Projection method to reduce internal forces}

For RA-PKM, the control torques may contain antagonistic forces that does not create any motion as they are in the null-space of $J_{m}^{T}$ and, thus, need to be removed. This can be achieved by projecting the input torques onto the range of $J_{m}^{T}$ by the following projector [6]: 


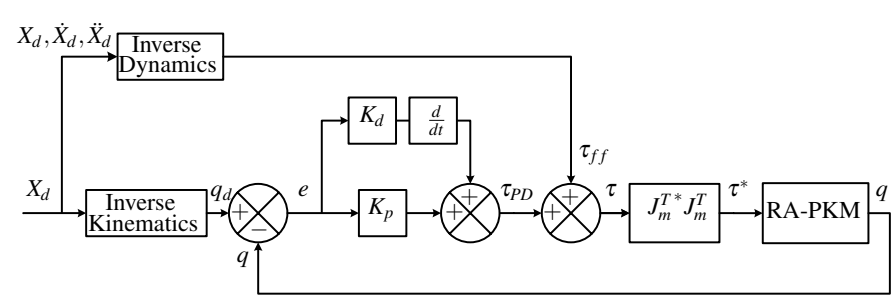

Fig. 5: Block diagram of the proposed control scheme

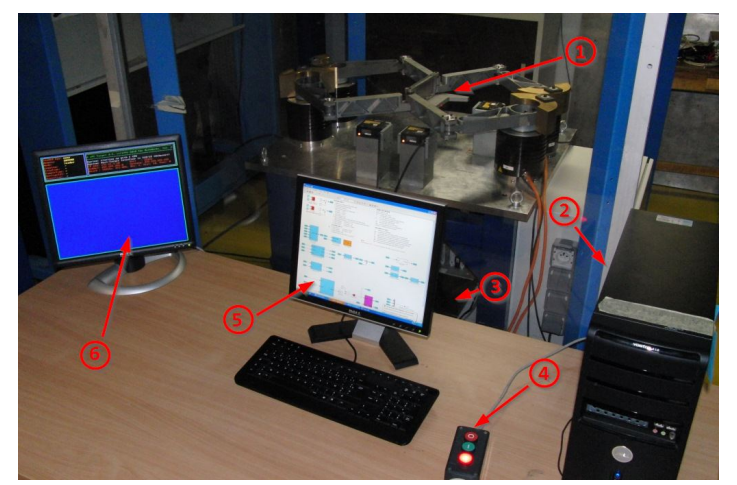

Fig. 6: View of the experimental setup; (1) the parallel manipulator, (2) the host PC, (3) the target PC, (4) emergency stop, (5) host monitor, (6) xPC target monitor

$$
R_{J_{m}^{T}}=I-N_{J_{m}^{T}}
$$

where $N_{J_{m}^{T}}=I-J_{m}^{T *} J_{m}^{T}$ is the projector to the null-space of $J_{m}^{T}$. Hence, the internal antagonistic forces are eliminated by projecting the control torques onto the range space of $J_{m}^{T}$. This is achieved by using the projection matrix $R_{J_{m}^{T}}$ as:

$$
\tau^{*}=R_{J_{m}^{T}}\left(\tau_{P D}+\tau_{f f}\right)
$$

The block diagram of the proposed control scheme is depicted in Fig 5.

\section{REAL-TIME EXPERIMENTAL RESULTS}

\section{A. The Dual-V robot and its hardware configuration}

The Dual-V links are all made with Aluminum and mounted on four direct drive actuators provided by ETEL Motion Technology. The actuators are fixed on an Aluminum base and can supply up to 127 N.m torques. We use Simulink and Real-Time Workshop (both from Mathworks Inc.) to implement and build the control algorithm. The generated code is then uploaded to the target PC; an industrial computer cadenced at $10 \mathrm{kHz}$ and running xPC Target in real-time. The experimental setup is depicted in Fig. 6.

\section{B. Real-time experimental results}

The proposed trajectory generator as well as the proposed controller presented in the previous section were implemented on the Dual-V robot. For comparison purpose, we also implement the classical approach using the original unmodified equations. In order to further investigate the benefits of the proposed approach, four different geometric shapes are implemented, namely; a circle, an ellipse, a tear-like and a deltoide (cf. Fig. 7a). The traveling plate of the manipulator has to perform every geometric trajectory in $T=0.5 \mathrm{~s}$. Between the end point of a shape trajectory and the starting point of the next one, the end-effector has to perform a PtP trajectory defined by a $5^{\text {th }}$ order polynomial function.

The controller feedback gains have been experimentally tuned using the trial-and-error technique. Usually, industrial robots are not equipped with velocity sensors, thus velocities have to be estimated. In our case, a carefully designed low-pass filter is used to estimate the velocities.

Figures $7 \mathrm{~b}$ and $7 \mathrm{c}$ depict the Cartesian tracking performance of the end-effector on both $x$ and $y$ axes. We can clearly see that in the case of original trajectories, the discontinuities cause a poor tracking performance. This is more noticeable at the beginning and the end of the circle and the ellipse. If the robot was equipped with accelerometers, one could notice the high frequency vibrations. However, if we check the obtained performance using the proposed approach, we can clearly see the improvements in terms of tracking errors at the end and starting points. It can be clearly seen that the proposed trajectories are accurately tracked thanks to the removed discontinuities. The improvements are more noticeable on the starting and end points of the circle and ellipse trajectories $(t=5,5.5,7.5$ and $8 s)$. These results were expected since the main purpose of this paper is to present a technique to remove these discontinuities from the trajectories generated by means of classical analytical functions. These discontinuities are a major source of tracking loss.

The control inputs generated when using the proposed method as well as those generated by classical one are depicted in Fig. 8. As expected, the controller generates very high torques when it faces discontinuities on the reference trajectories, because the drives need to generate high torques in order step from zero to a certain velocity in a very short time, which is practically very delicate.

\section{CONCLUSIONS AND FUTURE WORK}

In this paper we investigated the continuous closed shape trajectories generation and the reduction of internal forces by means of control for RA-PKMs. These trajectories are defined using analytical functions that show inherent discontinuities. For this reason, we proposed in this work an approach to overcome this inconvenience for a specific class of trajectories by modifying the time function without changing the overall closed shape of the trajectory in operational space. Furthermore, to overcome the phenomenon of internal forces, a PD with computed feedforward controller has been implemented. The resulting control torques have been projected using the Jacobian matrix to reduce internal forces that can harm the mechanical structure. The latter helps in significantly reducing the energy consumption and the vibrations on the robot's traveling plate. To validate the proposed approaches, realtime experiments have been conducted on a real 3 dof planar redundantly actuated parallel manipulator developed in our laboratory. The proposed trajectory generator exhibited its superiority over the original trajectory generator that utilizes original analytical equations. 


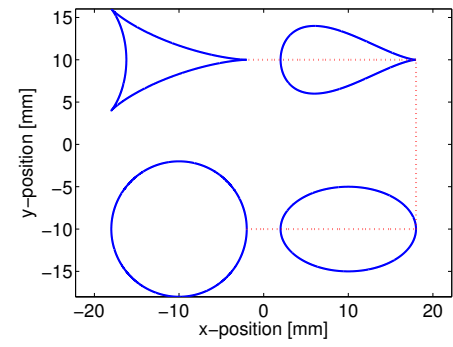

(a) Reference trajectory

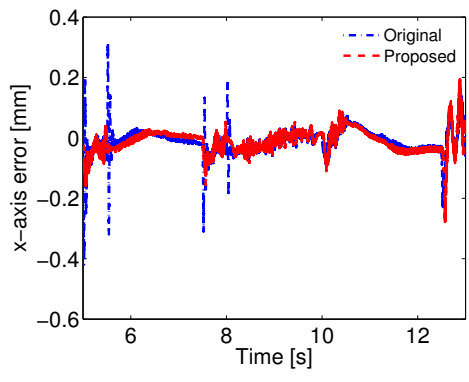

(b) Error on $x$ axis

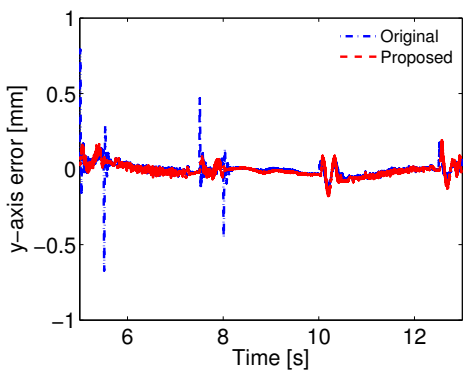

(c) Error on $y$ axis

Fig. 7: Reference trajectory and evaluation of the Cartesian tracking errors versus time
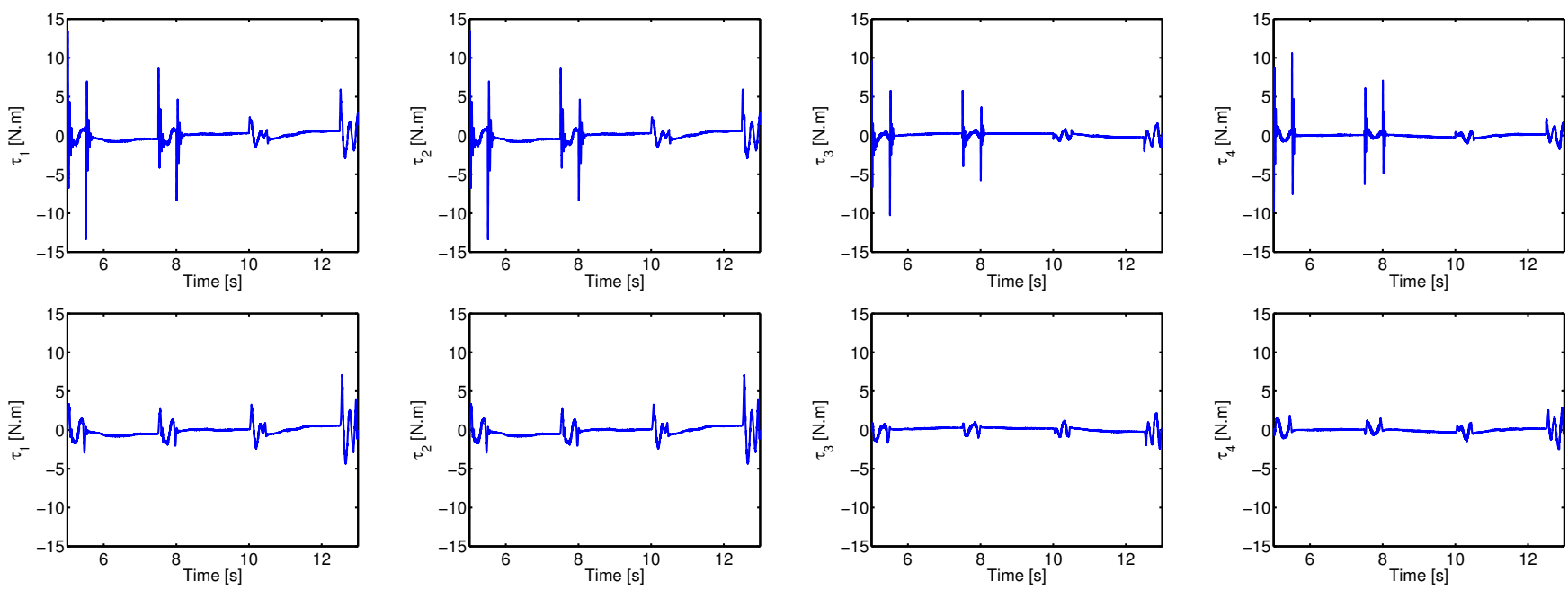

Fig. 8: Evolution of control torques: (top) original discontinuous trajectories and (bottom) proposed continuous trajectories

\section{REFERENCES}

[1] Hui Cheng, Yiu, Y.-K., and Zexiang Li., Dynamics and Control of Redundantly Actuated Parallel Manipulators, Transactions on Mechatronics, vol. 8, no. 4, pp. 483-491, 2003.

[2] Wijk, V. van der, Krut, S., Pierrot, F., and Herder, J. L., Generic Method for Deriving the General Shaking Force Balance Conditions of Parallel Manipulators with Application to a Redundant Planar 4-RRR Parallel Manipulator, The 13th World Congress in Mechanism and Machine Science, 2011

[3] Corbel, D., Gouttefarde, M., and Company, O, Towards 100G with PKM. Is Actuation Redundancy a Good Solution for Pick and Place? International Conference on Robotics and Automation, pp. 4675-4682, 2010.

[4] Liu, Y., Wang, C., Li, J., and Sun, L., Time-Optimal Trajectory Generation of a Fast-Motion Planar Parallel Manipulator, International Conference on Intelligent Robots and Systems (IROS'06), pp. 754-759, 2006.

[5] Macfarlane, S., and Croft, E. A., Jerk-bounded manipulator trajectory planning: design for real-time applications, IEEE Transactions on Robotics and Automation, vol. 19, no. 1, pp. 42-52, 2003.

[6] Miiller, A., and Hufnagel, T., A projection method for the elimination of contradicting control forces in redundantly actuated PKM, International Conference on Robotics and Automation, pp. 3218-3223, 2011.

[7] Wu, J., Wang, J., and You, Z., A comparison study on the dynamics of planar 3-DOF 4-RRR, 3-RRR and 2-RRR parallel manipulators, Robotics and Computer Integrated Manufacturing, vol. 27, no. 1, pp. 150-156, 2011.

[8] van der Wijk, S. Krut, F. Pierrot, and J. L. Herder, Design and exper- imental evaluation of a dynamically balanced redundant planar 4-RRR parallel manipulator, The International Journal of Robotics Research, vol. 32, no. 6, pp. 744-759, 2013.

[9] Merlet JP, Parallel Robots, Norwell, MA: Kluwer, 2000.

[10] Bonev, I., Delta parallel robot-the story of success, Newsletter, 2001.

[11] Su, H. J., Dietmaier, P., and McCarthy, J. M., Trajectory planning for constrained parallel manipulators. Journal of mechanical design, vol. 125, no. 4, pp. 709, 2003.

[12] Bruzzone, L. E., Molfino, R.M and Razzoli, R.P, Modeling and design of a parallel robot for laser-cutting applications, International Conference on Modeling, Identification and Control (IASTED’02), pp. 518-522, 2002.

[13] Chanal, H., Etude de l'emploi des machines outils structure parallle en usinage (Doctoral dissertation, Universit Blaise Pascal-Clermont-Ferrand II), 2006.

[14] Paccot, F., Andreff, N., and Martinet, P., A Review on the Dynamic Control of Parallel Kinematic Machines: Theory and Experiments, The International Journal of Robotics Research, vol. 28, no. 3, pp. 395-416, 2009.

[15] He, J. F., Jiang, H. Z., Cong, D. C., Ye, Z. M., and Han, J. W., A survey on control of parallel manipulator, Key Engineering Materials, vol. 339, pp. 307-313, 2007.

[16] Reyes, F. and Kelly, R., Experimental evaluation of model-based controllers on a direct-drive robot arm, Mechatronics, Vol. 11, no 3, pp. 267-282, 2001. 